\title{
On Traces in Some Analytic Spaces in Bounded Strictly Pseudoconvex Domains
}

\author{
Romi F. Shamoyan ${ }^{1}$ and Olivera R. Mihić ${ }^{2}$ \\ ${ }^{1}$ Bryansk State University, Bryansk 241050, Russia \\ ${ }^{2}$ Fakultet Organizacionih Nauka, Jove Ilića 154, 11000 Belgrade, Serbia \\ Correspondence should be addressed to Olivera R. Mihić; oliveradj@fon.rs
}

Received 23 November 2014; Accepted 28 January 2015

Academic Editor: Alberto Fiorenza

Copyright ( 2015 R. F. Shamoyan and O. R. Mihić. This is an open access article distributed under the Creative Commons Attribution License, which permits unrestricted use, distribution, and reproduction in any medium, provided the original work is properly cited.

New sharp estimates of traces of Bergman type spaces of analytic functions in bounded strictly pseudoconvex domains are obtained. These are, as far as we know, the first results of this type which are valid for any bounded strictly pseudoconvex domains with smooth boundary.

\section{Introduction and Formulation of Problem}

In this note we obtain new sharp estimates for traces in Bergman type spaces of analytic spaces in strictly pseudoconvex domains with smooth boundary. This line of investigation can be considered as a continuation of our previous papers on traces in analytic function spaces [1-4] where similar results were obtained but only in simpler bounded domains in higher dimension. We remark that in this note for the first time in the literature we consider this known problem related with trace estimates in spaces of analytic functions in more general pseudoconvex domains in $\mathbb{C}^{n}$, namely, in strictly pseudoconvex domains with smooth boundary. The next section contains required preliminaries on analysis on bounded strictly pseudoconvex domains with smooth boundary. Our new sharp results are contained in the last section of this note. Related estimates for Bergman type projections will be also provided. All our main results in context of unit ball can be seen in [2-4]. All preliminary assertions of this paper have their direct analogues in context of unit ball and this can be seen in [2-4] and references therein. It is known that the geometry of pseudoconvex domains is more complicated and extra arguments were needed to get technical lemmas which are used to prove main results of this paper. These subtle lemmas can be seen in particular in recent papers $[5,6]$.
Now we will shortly present the history of the diagonal map (or traces) problem. After the appearance of [7], various papers appeared where arguments which can be seen in [7] were extended, changed, and modified in various directions in one and higher dimension (see, e.g., $[1,3,8,9]$ and also various references therein). In particular in mentioned papers various new sharp results on traces for analytic function spaces in higher dimension (unit polyball) were obtained. New results for large scales of analytic $Q_{p}$ type spaces in polyball were proved (see [4]). Later several new sharp results for harmonic functions of several variables in the unit ball and upper half-plane of Euclidean space were also obtained (see, e.g., [1] and references therein). For the first time in the literature, these types of problems connected with diagonal map in analytic spaces appeared before in [7]. In [7], this problem was formulated and certain concrete cases connected with spaces of analytic functions in the unit disk were considered.

Some interesting applications of diagonal map can be seen in $[8,10]$ where other problems around this topic can be found also. The goal of this note is to develop further some ideas from our recent mentioned papers and present new sharp theorems in strictly pseudoconvex domains with smooth boundary. 
Extension problems were studied mainly by two different methods. The one is the extension using integral formula in the case where $D$ is bounded pseudoconvex domains with a support function (domains with smooth boundary). The other is the $L^{2}$ extension using the Hilbert space theory in the case when $D$ is general bounded pseudoconvex domain (see [11-14]).

For formulation of our results, we will need various standard definitions from the theory of strictly pseudoconvex domains with smooth boundary. In this and next section, we mention some vital facts which will be heavily used in proofs of our assertions (see, e.g., also for parallel assertion in other domains [2-4]).

Let $D=\{z: \rho(z)<0\}$ be a bounded strictly pseudoconvex domain of $\mathbb{C}^{n}$ with $C^{\infty}$ boundary. We assume that the strictly plurisubharmonic function $\rho$ is of class $C^{\infty}$ in a neighborhood of $\bar{D}$; that is, $-1 \leq \rho(z)<0, z \in D,|\partial \rho| \geq$ $C_{0}>0$ for $|\rho| \leq r_{0}$. Let further $H(D)$ be the space of all analytic functions on $D$.

Let (see [15])

$$
A_{\delta, k}^{p, q}(D)=\left\{f \in H(D):\|f\|_{p, q, \delta, k}<\infty\right\},
$$

where

$$
\|f\|_{p, q, \delta, k}=\left(\sum_{|\alpha| \leq k} \int_{0}^{r_{0}}\left(\int_{\partial D_{r}}\left|D^{\alpha} f\right|^{p} d \sigma_{r}\right)^{q / p} r^{\delta q / p-1} d r\right)^{1 / q},
$$

where $D_{r}=\left\{z \in \mathbb{C}^{n}: \rho(z)<-r\right\}, \partial D_{r}$ is boundary, $d \sigma_{r}$ is the normalized surface measure on $\partial D_{r}$ and by $d r$ normalized volume element on $(0, r), 0<p<\infty, 0<q \leq \infty, \delta>0$, $k=0,1,2, \ldots$, and

$$
\begin{aligned}
& \|f\|_{p, \infty, \delta, k} \\
& \quad=\sup \left\{\left(\sum_{|\alpha| \leq k} r^{\delta} \int_{\partial D_{r}}\left|D^{\alpha} f\right|^{p} d \sigma_{r}\right)^{1 / p}: 0<r<r_{0}\right\},
\end{aligned}
$$

where $D^{\alpha}$ is a differential operator acting from $H(D)$ to $H(D)$ (see [15] and references therein for this operator and it is properties). For $p, q<1$, it is quasinorm (see [15]). For $p=q$,

$$
\|f\|_{p, \delta, k}=\left(\sum_{|\alpha| \leq k} \int_{D}\left|D^{\alpha} f\right|^{p}(-\rho)^{\delta-1} d m(\xi)\right)^{1 / p}
$$

where $d m$ is the Lebesgue measure on the $D$ domain. We listed some properties of these classes below in Lemma C. $[5,6])$.

For $p=q, k=0$, we get Bergman spaces $A_{\delta}^{p}(D)$ (see

Let $B_{D}(z, r)$ be Kobayashi ball in bounded pseudoconvex domain (see $[5,6]), \delta(w): D \rightarrow \mathbb{R}^{+}$, and $\delta(w)=d(w, \partial D)$.

One of the intentions of this paper is to consider new trace problem and trace map for this case of bounded strictly pseudoconvex domains with smooth boundary. It is a map
$\operatorname{Tr} f(z)=f(z, \ldots, z), z \in D$, if $f \in X \subset H\left(D^{m}\right)$ for certain quasinormed space $X$ on $m$ products of $D$ domains $D^{m}$, where $H\left(D^{m}\right)$ is a space of analytic functions in products domains $D^{m}$.

Note these type maps were previously considered by various authors in particular cases when $\Omega=\mathbf{D}$ (unit disc), when $\Omega=\mathbf{B}$ (unit ball). Applications of this map to various problems in function theory are also known (see, e.g., [2, 4, 10] and references therein).

Note in addition that we use heavily the same machinery which was recently developed in $[5,6]$.

The trace problem in particular is in short the following. Let $f \in H\left(D^{m}\right), D^{m}=D \times \cdots \times D$ and $f$ satisfies certain growth condition $f \in X, X \subset H\left(D^{m}\right)$ and then get as much information as possible about growth of $f(z, \ldots, z), z \in D$, where $X$ can be certain fixed functional class of analytic functions $f\left(z_{1}, \ldots, z_{m}\right), z_{i} \in D, i=1, \ldots, m$ (analytic by each variable). We also will look at the same time at estimates of various multifunctional operators and expressions closely related with restriction map.

The technique we use is based also on a work of Beatrous (see [12]) and Ortega-Fabrega (see [15]) and some information from $[5,6]$ on pseudoconvex domains (namely, some subtle estimates from very recent papers $[5,6]$ ) will be also used. Note that various similar extension theorems were previously studied by many authors (Henkin, Adachi, and Cumenge); see [11-13, 15] and various references therein.

Actually in this paper we continue (partially) the investigation of Jimbo and Sakai (see [16]) related to function spaces on products of pseudoconvex domains in $\mathbb{C}^{n}$.

To define new Bergman type analytic spaces on products of pseudoconvex domains we have to replace one integral by multiple integrals in spaces above. For example, the mentioned quasinorms will take this form:

$$
\begin{aligned}
& \|f\|_{p, \delta_{1}, \ldots, \bar{\delta}_{m}, k_{1}, \ldots, k_{m}} \\
& =\|f\|_{p, \vec{\delta}, \vec{k}} \\
& =\left(\sum_{\substack{\left|\alpha_{1}\right| \leq k_{1} \\
\left|\alpha_{m}\right| \leq k_{m}}} \int_{D} \cdots \int_{D}\left|D^{\alpha_{1} \cdots \alpha_{n}} f\left(z_{1}, \ldots, z_{m}\right)\right|^{p} \cdot(-\rho)^{\delta_{1}-1}\right. \\
& \left.\cdots(-\rho)^{\delta_{m}-1} d m\left(\xi_{1}\right) \cdots d m\left(\xi_{m}\right)\right)^{1 / p},
\end{aligned}
$$

where $f \in H\left(D^{m}\right)$ and $\delta_{j}>0, j=1, \ldots, m$.

We formulate in the next section some new results related to restriction maps in products of pseudoconvex domains and multifunctional spaces generalizing previous estimates for polydisk (when $D$ is a unit disk). Note now our goal 
in this paper to study only particular case of general $A_{\delta, k}^{p, q}$ classes and integral operators (Bergman type) on them. Note also that Bergman type projection from various points of view in pseudoconvex domains was studied before via various authors (see, e.g., [12, 15, 17-19] and references therein).

The study of traces of general $A_{\delta, k}^{p, q}$ spaces (including limit case) is a separate problem which will be addressed by authors in a separate paper.

The study of analytic spaces in products of pseudoconvex domains was started probably in $[16,20]$. This paper is heavily based on arguments that appeared in proofs of results from $[2,4]$. Using them, we here extend various assertions which can be found in unit ball in $[2,4]$ to the case of general strictly pseudoconvex bounded domains $D$ with smooth boundary.

Throughout the paper $C$, sometimes with indexes, stands for various positive constants which can be different even in a chain of inequalities and are independent of the discussed functions or variables.

The notation $A \asymp B$ means that there is a positive constant $C$, such that $B / C \leq A \leq C B$. We will write for two expressions $A \lesssim B$ if there is a positive constant $C$ such that $A<C B$.

\section{Preliminaries and Formulations of Main Theorems}

In this section, we collect preliminaries and formulations of all main results of this paper.

We define Bergman spaces on polypseudoconvex domains as

$$
\begin{aligned}
& A_{\vec{\beta}}^{p}\left(D^{m}\right) \\
& =A^{p}\left(D^{m}, \vec{\beta}\right) \\
& =\{f \in H(D \times \cdots \times D): \\
& \quad \int_{D} \cdots \int_{D}\left|f\left(w_{1}, \ldots, w_{m}\right)\right|^{p} \delta\left(w_{1}\right)^{\beta_{1}} \\
& \quad<\infty\},
\end{aligned}
$$

where $d m$ is the Lebesgue measure on the $D$ domain, $0<p<$ $\infty$, and $\beta_{j}>-1, j=1, \ldots, m$. These are Banach spaces for $1<p<\infty$ and complete metric spaces for $0<p \leq 1$. We put $d m_{s}(z)=\delta^{s}(z) d m(z), s>-1$.

Let $\operatorname{Trace}(X)=\left\{f(z, \ldots, z): f \in X \subset H\left(D^{m}\right)\right\}$, where $X$ is quasinormed space in product domains. Theorem 1 is one of the main results of this paper.

Theorem 1. Let $p \in(0, \infty)$, and $\beta_{i}>-1, i=1, \ldots, m$. Then,

$$
\operatorname{Trace}^{p}\left(D^{m}, \vec{\beta}\right) \subset A^{p}\left(D, \sum_{i=1}^{m} \beta_{i}+(m-1)(n+1)\right) \text {. }
$$

The proof of this theorem is based only on Propositions 3 and 4. It is completely parallel to the case of unit ball, which was considered before as in $[2,4]$. We omit it now. We start with covering lemma (then other four lemmas) for $D$ by using Kobayashi balls $B_{D}(z, r)$ (see $\left.[5,6]\right)$, but first we need Definition 2.

Definition 2 (see $[5,6]$ ). Let $D \subset \mathbb{C}^{n}$ be a bounded domain and $r>0$. An $r$-lattice in $D$ is a sequence $\left\{a_{k}\right\} \subset D$ such that $D=\bigcup_{k} B_{D}\left(a_{k}, r\right)$ and there exists $m>0$ such that any point in $D$ belongs to at most $m$ balls of the form $B_{D}\left(a_{k}, R\right)$, where $R=(1 / 2)(1+r)$.

Proposition 3 (see $[5,6]$ ). Let $D \subset \mathbb{C}^{n}$ be a bounded strictly pseudoconvex domain with smooth boundary. Then for every $r \in(0,1)$ there exists an $r$-lattice in $D$; that is, there exists $m \in \mathbb{N}$ and a sequence $\left\{a_{k}\right\} \subset D$ of points such that $D=$ $\bigcup_{k=0}^{\infty} B_{D}\left(a_{k}, r\right)$ and no point of $D$ belongs to more than $m$ of the balls $B_{D}\left(a_{k}, R\right)$, where $R=(1 / 2)(1+r)$.

Proposition 4 (see $[5,6])$. Let $D \subset \mathbb{C}^{n}$ be a bounded strictly pseudoconvex domain with smooth boundary. Given $r \in(0,1)$, set $R=(1 / 2)(1+r) \in(0,1)$. Then, there exists a $k_{r}>0$ depending on $r$ such that for all $z_{0} \in D$ and for all $z \in B_{D}\left(z_{0}, r\right)$

(1) $f(z) \leq\left(k_{r} / m\left(B_{D}\left(z_{0}, r\right)\right)\right) \int_{B_{D}\left(z_{0}, r\right)}(f(w)) d m(w)$,

(2) $m\left(B_{D}(\cdot, r)\right) \approx \delta^{n+1}$,

for every $f$-nonnegative plurisubharmonic function $f: D \rightarrow$ $\mathbb{R}^{+}$.

Let now $D$ be a $C^{\infty}$-bounded strongly pseudoconvex domain with defining function $\rho$. We need some results for our proofs. We let $g(z, \psi)$ be the associated Levi polynomial (see [21]). Consider

$$
\begin{aligned}
g(z, \zeta)= & 2 \sum_{j=1}^{n} \frac{\partial \rho}{\partial \zeta_{j}}(\zeta)\left(\zeta_{j}-z_{j}\right) \\
& -\sum_{j, k=1}^{n} \frac{\partial^{2} \rho}{\partial \zeta_{j} \partial \zeta_{k}}(\zeta)\left(\zeta_{j}-z_{j}\right)\left(\zeta_{k}-z_{k}\right) .
\end{aligned}
$$

It follows from Taylor's formula and the strict plurisubharmonicity of $\rho$ that there are positive constants $C_{1}$ and $r$ and a neighborhood $D^{\prime}$ of $\bar{D}$ such that

$$
\operatorname{Re} g(z, \zeta) \geq \rho(\zeta)-\rho(z)+C_{1}|z-\zeta|^{2}
$$

for $z, \zeta \in D^{\prime}$ and $|z-\zeta| \leq r$. Setting $\tilde{g}(z, \zeta)=g(z, \zeta)-2 \rho(\zeta)$, it follows that

$$
\begin{aligned}
\operatorname{Re} \tilde{g}(z, \zeta) & =\operatorname{Re} g(z, \zeta)-2 \rho(\zeta) \\
& \geq-\rho(\zeta)-\rho(z)+C_{1}|z-\zeta|^{2}
\end{aligned}
$$


for $z, \zeta \in D^{\prime}$ and $|z-\zeta| \leq r$ and $\widetilde{g}(z, \zeta)=g(z, \zeta)$ for $\zeta \in \partial D$. Also we have

$$
\mathcal{N} \widetilde{g}(z, \zeta)=\mathscr{O}\left(|z-\zeta|^{2}\right),
$$

where $\mathcal{N}$ is the complex normal vector field of type $(1,0)$ defined by $\mathcal{N}=\sum_{j=1}^{n}\left(\partial \rho / \partial \bar{\zeta}_{j}\right)\left(\partial / \partial \zeta_{j}\right)$.

Lemma A (see [21]). Let $\widetilde{g}, D^{\prime}, r$, and $C_{1}$ be as above. There is a neighborhood $\widetilde{D}$ of $\bar{D}$ with $\widetilde{D} \supset \bar{D}, a C^{\infty}$ function $\widetilde{\Phi}$ on $\widetilde{D} \times \widetilde{D}$, and a positive constant $C_{2}$ such that

(1) for any $\zeta \in \widetilde{D}$ the function $\widetilde{\Phi}(\cdot, \zeta)$ is holomorphic on $\widetilde{D}$;

(2) $\widetilde{\Phi}(\zeta, \zeta)=-2 \rho(\zeta)$ for $\zeta \in \widetilde{D}$ and $|\widetilde{\Phi}(z, \zeta)| \geq C_{2}$ for $z, \zeta \in \widetilde{D}$ with $|z-\zeta| \geq r / 2$

(3) there is a nonvanishing $C^{\infty}$ function $Q(z, \zeta)$ on

$$
\Delta_{r / 2}=\left\{(z, \zeta) \in \widetilde{D} \times \widetilde{D}:|z-\zeta| \leq \frac{r}{2}\right\}
$$

such that

$$
\widetilde{\Phi}(z, \zeta)=\widetilde{g}(z, \zeta) Q(z, \zeta) \quad \text { on } \Delta_{r / 2} .
$$

Lemma B (see [21]). For each $s>-1$, there is a smooth form $\eta_{s} \in C^{\infty}(\widetilde{D} \times \widetilde{D})$ such that

(1) $\eta_{s}(z, \zeta)$ is holomorphic in $z$ on $\widetilde{D}$ for any fixed $\zeta \in \widetilde{D}$ and

(2) for $f \in A_{s}^{1}(D)$ and $z \in D$ one has

$$
f(z)=\int_{D} f(\zeta) \frac{\eta_{s}(z, \zeta)}{\widetilde{\Phi}(z, \zeta)^{n+s+1}}(-\rho(\zeta))^{s} d m(\zeta) .
$$

Definition 5 (see [12]). Let $K(z, \xi)$ be a measurable function on $\bar{D} \times \bar{D}$ and let $t$ be a positive number. One says that $K=K_{t}$ (or $\widetilde{K}_{t}$ ) is a kernel of Bergman type $t$ for all $z \in D$, if $|K(z, \xi)| \leq$ $C\left(|\widetilde{\Phi}(z, \xi)|^{-t}\right)$ where $\widetilde{\Phi}$ is Henkin-Ramirez function. So, if $K$ is a kernel of Bergman type $t$, then $K^{s}$ is a kernel of type st.

In this paper we deal with the following kernel $K_{n+1+t}(z$, $w)$ of $n+t+1$ type which is the reproducing Bergman kernel for weighted Bergman spaces in pseudoconvex domains with smooth boundary (see $[12,22])$.

We need also estimates for Bergman type kernel, the socalled Forelli-Rudin type estimates. The following assertion is valid if we change the index of kernel $n+1$ to $n+t+1$ (and $(n+1)(p-1)$ to $(n+1+t) p-(n+1)$ in other places; see [22]).

Proposition 6 (see $[5,6])$. Let $D \subset \mathbb{C}^{n}$ be a bounded strictly pseudoconvex domain with smooth boundary and let $z_{0} \in D$ and $1 \leq p<\infty$. Then, one has the following estimate for Bergman type kernel $K_{n+1}\left(z, z_{0}\right)$ :

$$
\begin{aligned}
& \int_{D}\left|K_{n+1}\left(\xi, z_{0}\right)\right|^{p} \cdot \delta(\xi)^{\beta} d m(\xi) \\
& \quad \lesssim \begin{cases}\delta\left(z_{0}\right)^{\beta-(n+1)(p-1)}, & -1<\beta<(n+1)(p-1), \\
\left|\log \delta\left(z_{0}\right)\right|, & \beta=(n+1)(p-1), \\
1, & \beta>(n+1)(p-1) .\end{cases}
\end{aligned}
$$

Estimate (15) is valid for all $K_{t}$ kernels (see [12, 22]). Let

$$
\begin{array}{r}
L_{s}^{p}(D)=\left\{f \in L^{\circ}(D):\|f\|_{p, s}^{p}=\int_{D}|f(w)|^{p} d m_{s}(w)<\infty\right\}, \\
p \geq 1, \quad s>-1 .
\end{array}
$$

Let us further define Bergman type integral operators. If $F \in H(D) \cap L_{s}^{2}(D)$, then we have Bergman type representation

$$
\begin{gathered}
F(z)=P_{1, s}(F)(z), \\
P_{s, m} F(\vec{z})=\int_{D} F(w) \prod_{i=1}^{m}\left(K_{(s+n+1) / m}\left(z_{i}, w\right)\right) d m_{s}(w),
\end{gathered}
$$

for all $z \in D, s>-1$. If $f \in L_{s}^{1}(D)$, we define the operator (Bergman type operator) for all $z_{j} \in D, j=1, \ldots, m$ :

$$
P_{s, m}(f)(\vec{z})=\int_{D} f(\xi) \prod_{i=1}^{m}\left(K_{(s+n+1) / m}\left(\xi, z_{i}\right)\right) d m_{s}(\xi) .
$$

Lemma C (see [12]). Let $f \in A_{t}^{1}(D)$ for sufficiently large t. Then,

$$
f(z)=\int_{D} f(\xi) K(z, \xi) \delta(\xi)^{t} d m(\xi),
$$

where $K(z, \xi)$ is a certain fixed kernel of $n+t+1$ type, $z \in D$.

Proposition 7 (see [12]). Let $f \in A_{\alpha}^{p}(D), 0<p<\infty, \alpha>-1$. Then, (19) integral representation holds with large enough $t$.

Proof. If $f \in A_{\alpha}^{p}(D)$, then for large enough $t$ we have $f \in$ $A_{t}^{1}(D)$. The proof of these facts follows from the well-known proof in the unit disk case. Then, use Lemma C.

We now also note that the following is true. Let

$$
\begin{aligned}
& \Phi\left(z_{1}, \ldots, z_{m}\right) \\
& =\int_{D} f(\xi) K_{(t+n+1) / m}\left(z_{1}, \xi\right) \cdots K_{(t+n+1) / m} \\
& \quad \cdot\left(z_{m}, \xi\right) \delta(\xi)^{t} d m(\xi)
\end{aligned}
$$

$z_{j} \in D, j=1, \ldots, m$. We have

$$
\begin{aligned}
\Phi(z, \ldots, z) & =\int_{D} f(\xi)\left(K_{(t+n+1) / m}(z, \xi)\right)^{m} \delta(\xi)^{t} d m(\xi) \\
& =\int_{D} f(\xi) \widetilde{K}_{t+n+1}(z, \xi) \delta(\xi)^{t} d m(\xi)=f(z)
\end{aligned}
$$

where $t>t_{0}, t_{0}$ is large enough, and $z \in D, f \in A_{\alpha}^{p}, p>0$, $\alpha>-1$. 
The study of $\Phi=T_{m, t} f$ operator (expanded Bergman projection) is of special interest. This operator was considered by many authors before in various situations (polydisk, unit ball, and spaces of harmonic functions in $\mathbb{R}_{+}^{n+1}$ and $\mathbf{B}$ ) and used in relation to traces problems (see $[2,4]$ and references therein).

We provide some new estimates here for expanded Bergman projection based on our fully previous work in unit ball extending known estimates for ordinary Bergman projection in strictly pseudoconvex domains in weighted Bergman $A_{\alpha}^{p}$ spaces. Our results again are heavily based on lemmas from $[5,6]$.

Theorem 8. Let $1 \leq p<\infty$ and let $\alpha_{j}>-1, j=1, \ldots, m$, and

$$
\begin{aligned}
& T_{m, t} f\left(z_{1}, \ldots, z_{m}\right) \\
& =\int_{D} f(\xi) \delta(\xi)^{m(n+t+1)-(n+1)} \\
& \cdot\left|\prod_{i=1}^{m} K_{n+t+1}\left(z_{j}, \xi\right)\right| d m(\xi),
\end{aligned}
$$

where $z_{j} \in D, j=1, \ldots, m$, and $t>t_{0}$ for large enough $t_{0}$. Then, we have

$$
\left\|T_{m, t} f\right\|_{A_{\alpha_{1}}^{p}} \leq C\|f\|_{A_{\alpha_{1}}^{p}},
$$

where $\alpha_{1}=\sum_{1}^{m} \alpha_{j}+(n+1)(m-1)$ and hence

$$
\begin{gathered}
\int_{D} \cdots \int_{D}\left|T_{m, t} f\left(z_{1}, \ldots, z_{m}\right)\right|^{p} \delta^{\alpha_{1}}\left(z_{1}\right) \\
\cdots \delta^{\alpha_{m}}\left(z_{m}\right) d m\left(z_{1}\right) \cdots d m\left(z_{m}\right) \\
\leq C \int_{D}|f(\xi)|^{p} \delta(\xi)^{\tau_{1}} d m(\xi), \\
\tau_{1}=\sum_{j=1}^{m} \alpha_{j}+(n+1)(m-1) .
\end{gathered}
$$

Remark 9. It will be interesting for reader to compare this result with Theorem 4.1 from [12] where similar integral operator with $K_{t}$ kernel was considered and used in proof of main results.

Remark 10. A variant of Theorem 8 is true also for $p \leq 1$. We will suggest comparing our proofs with Corollary 5.3 of [12] where $m=1$ case was considered.

Remark 11. From Proposition 7 we have, for large enough $t, f \in A_{\alpha}^{p}$, and if we define

$$
\begin{aligned}
\widetilde{\Phi}\left(z_{1}, \ldots, z_{m}\right)=\int_{D} f(\xi) K_{(t+n+1) / m}\left(z_{1}, \xi\right) \\
\\
\cdots K_{(t+n+1) / m}\left(z_{m}, \xi\right) \delta(\xi)^{t} d m(\xi),
\end{aligned}
$$

then $\widetilde{\Phi}(z, \ldots, z)=f(z)$; since the integral representation (19) is valid for large enough $t, f \in A_{t}^{1}$. This together with assertion concerning the action of $\widetilde{\Phi}\left(z_{1}, \ldots, z_{m}\right)$ from Theorem 8 gives another theorem on traces (see proof below).

Theorem 12. Let $f \in A^{p}\left(D^{m}, \vec{\beta}\right)$ and $\beta_{j}>-1,1 \leq p<\infty$, $j=1, \ldots, m$. Then,

$$
\operatorname{Trace}^{p}\left(D^{m}, \vec{\beta}\right)=A^{p}\left(D, \sum_{i=1}^{m} \beta_{i}+(m-1)(n+1)\right) .
$$

Remark 13. Note that a trace theorem can be extended to some mixed norm classes defined like this

$$
\begin{aligned}
A^{\vec{p}} & \left(D^{m}, \vec{\beta}\right) \\
= & \left\{f \in H\left(D^{m}\right):\right. \\
& \left.\int_{D}\left(\int_{D}\left(\int_{D}|f(\vec{w})|^{p_{1}} \delta\left(w_{1}\right)^{\beta_{1}} d m\left(w_{1}\right)\right)\right)^{p_{2} / p_{1}}<\infty\right\},
\end{aligned}
$$

where $0<p_{i}<\infty, \beta_{j}>-1, j=1, \ldots, m$ (see $[2,4]$ for unit ball case).

We formulate now new two trace theorems concerning $p=\infty$ case and $A^{p}\left(D^{m}, \vec{\beta}\right), p \leq 1$ spaces. Then, we will also below look at following operators following our papers $[2,4]$. Let $f \in L^{1}\left(D^{m}\right)$ and

$$
\begin{array}{r}
G_{\vec{x}, \vec{y}}(f)(\xi)=\int_{D} \cdots \int_{D} f\left(z_{1}, \ldots, z_{m}\right) \delta\left(z_{1}\right)^{x_{1}} \cdots \delta\left(z_{m}\right)^{x_{m}} \\
\cdot \prod_{j=1}^{m} K_{j}\left(z_{j}, \xi\right) d m\left(z_{1}\right) \cdots d m\left(z_{m}\right),
\end{array}
$$

where $K_{i}$ is a kernel of $x_{i}+n+1$ type, $i=1, \ldots, m$, and $\xi \in D$. Let also

$$
\begin{aligned}
& A_{\vec{r}}^{\infty}\left(D^{m}\right) \\
& =\left\{f \in H\left(D^{m}\right):\right. \\
& \begin{array}{l}
\sup _{z_{1} \in D}\left|f\left(z_{1}, \ldots, z_{m}\right)\right| \\
\vdots \\
z_{m} \in D
\end{array}
\end{aligned}
$$

$$
\left.\cdot \delta\left(z_{1}\right)^{r_{1}} \cdots \delta\left(z_{m}\right)^{r_{m}}<\infty\right\}
$$

$$
A_{r}^{\infty}(D)=\left\{f \in H(D): \sup _{z \in D}|f(z)| \delta(z)^{r}<\infty\right\} .
$$


Theorem 14. Let $r_{j}>0, j=1, \ldots, m, r=\sum_{j=1}^{m} r_{j}$. Then,

(1) $\operatorname{Trace}\left(A_{\vec{r}}^{\infty}\left(D^{m}\right)\right)=A_{r}^{\infty}(D)$,

(2) $\operatorname{Trace}\left(A_{\vec{\beta}}^{p}\left(D^{m}\right)\right)=A_{t}^{p}(D)$,

where $p \leq 1, \beta_{j}>-1, j=1, \ldots, m$, and $t=(m-1)(n+1)+$ $\sum_{j=1}^{m} \beta_{j}$.

The second part of the following theorem generalizes partially Theorem 8 .

Theorem 15 (on expanded Bergman projections). (1) Let $1 \leq$ $p<\infty, s_{j}>-1, j=1, \ldots, m$. Let also $x_{j}>a_{0}, y_{j}>b_{0}$, $a_{0}=a_{0}\left(p, s_{j}, n, m\right)$, and $b_{0}=b_{0}\left(p, s_{j}, n, m\right)$, where $a_{0}$ and $b_{0}$ are large enough, $j=1, \ldots, m$. Then there is a constant $C$ so that we have

$$
\begin{gathered}
\int_{D}\left|G_{\vec{x}, \vec{y}} f(\xi)\right|^{p}(\delta(\xi))^{(m-1)(n+1)+\sum_{j=1}^{m} s_{j}} d m(\xi) \\
\leq C \int_{D} \cdots \int_{D}\left|f\left(z_{1}, \ldots, z_{m}\right)\right|^{p} \\
\cdot \prod_{j=1}^{m}\left(\delta\left(z_{j}\right)\right)^{s_{j}} d m\left(z_{j}\right) .
\end{gathered}
$$

(2) Let

$$
\begin{gathered}
S_{\vec{a}, \vec{b}}(f)\left(\xi_{1}, \ldots, \xi_{m}\right) \\
=\prod_{i=1}^{m}\left(\delta\left(\xi_{i}\right)\right)^{\widetilde{a}_{i}} \int_{D} f(w)(\delta(w))^{-n-1+\sum_{j=1}^{m} b_{j}} \widetilde{K_{1}}\left(\xi_{1}, w\right) \\
\cdots \widetilde{K_{m}}\left(\xi_{m}, w\right) d m(w),
\end{gathered}
$$

where $\widetilde{K}_{j}$ is a kernel of type $a_{j}+b_{j}, j=1, \ldots, m$. Then, for $1 \leq p<\infty, s_{j}>-1$,

$$
\begin{gathered}
\int_{D} \cdots \int_{D}\left|S_{\vec{a}, \vec{b}}(f)\left(\xi_{1}, \ldots, \xi_{m}\right)\right|^{p} \prod_{j=1}^{m}\left(\delta\left(\xi_{j}\right)\right)^{s_{j}} d m\left(\xi_{j}\right) \\
\leq C \int_{D}|f(w)|^{p}(\delta(w))^{(m-1)(n+1)+\sum_{j=1}^{m} s_{j}} d m(w),
\end{gathered}
$$

where $a_{j}>a_{0}$ and $b_{j}>b_{0}$ for some large enough $a_{0}$ and $b_{0}$, $j=1, \ldots, m$.

(3) Let $p \leq 1, s_{j}>-1, j=1, \ldots, m$. Then,

$$
\begin{aligned}
& \int_{D} \cdots \int_{D}\left|S_{\vec{a}, \vec{b}}(f)\left(z_{1}, \ldots, z_{m}\right)\right|^{p} \prod_{j=1}^{m}\left(\delta\left(z_{j}\right)\right)^{s_{j}} d m\left(z_{j}\right) \\
& \quad \leq C\|f\|_{A_{(m-1)(n+1)+\sum_{j=1}^{m} s_{j}}^{p}}^{p}
\end{aligned}
$$

where $a_{j}>a_{0}$ and $b_{j}>b_{0}$ for some large enough $a_{0}$ and $b_{0}$, $j=1, \ldots, m$.
Exact values of $a_{0}=a_{0}(p, s, n, m)$ and $b_{0}=b_{0}(p, s, n, m)$ can be calculated as in unit ball case (see [3]).

Remark 16. Exact values of $x_{0}$ and $y_{0}$ in Theorem 15 can be calculated by readers. We refer the reader to unit ball case where details can be seen (see [2-4]).

Remark 17. The proof of Theorem 15 will be omitted by us. In the unit ball it can be seen in $[2,3,16]$. Moreover, the proof is based on same ideas as the proof of Theorems 1-14 with small modifications.

\section{Proofs of Theorems 1-15 and Final Comment}

In this section we provide proofs of all our main assertions which we formulated in this paper in the previous section.

Proof of Theorem 1. We have the following chain of estimates using properties of Kobayashi balls and Propositions 3-4:

$$
\begin{gathered}
J \int_{D}|f(w, \ldots, w)|^{p}(\delta(w))^{(m-1)(n+1)+\sum_{j=1}^{m} s_{j}} d m(w) \\
\leq C_{1} \sum_{k \geq 0} \int_{B_{D}\left(a_{k}, r\right)}|f(w, \ldots, w)|^{p} \\
\cdot(\delta(w))^{(m-1)(n+1)+\sum_{j=1}^{m} s_{j}} d m(w) \\
\leq C_{2} \sum_{k \geq 0}\left(\sup _{w \in B_{D}\left(a_{k}, r\right)}|f(w, \ldots, w)|^{p}\right) \\
\cdot \int_{B_{D}\left(a_{k}, r\right)}(\delta(w))^{(m-1)(n+1)+\sum_{j=1}^{m} s_{j}} d m(w) \\
=C_{3} \sum_{k \geq 0}\left(\sup _{w \in B_{D}\left(a_{k}, r\right)}|f(w, \ldots, w)|^{p}\right) \\
\cdot\left(\delta\left(a_{k}\right)\right)^{t} \cdot C(r) \cdot\left(\delta\left(a_{k}\right)\right)^{n+1}
\end{gathered}
$$

where $t=(m-1)(n+1)+\sum_{j=1}^{m} s_{j}$ and $C(r)$ is constant (depending on $r$ ). These estimates follow from Lemmas 2.1 and 2.2 of [5] which say

$$
\begin{gathered}
m\left(B_{D}(\cdot, r)\right) \approx \delta^{n+1}, \quad r \in(0,1), \\
\delta(z) \in\left[\frac{1-r}{\widetilde{C}} \cdot \delta\left(z_{0}\right), \frac{\widetilde{C}}{1-r} \cdot \delta\left(z_{0}\right)\right], \\
r \in(0,1), \quad z_{0} \in D, \quad z \in B_{D}\left(z_{0}, r\right) .
\end{gathered}
$$

From (34) we have using Propositions 3-4 finally

$$
\begin{aligned}
J \leq C \sum_{k_{1} \geq 0} \cdots \sum_{k_{m} \geq 0}\left(\begin{array}{l}
\sup _{w_{1} \in B_{D}\left(a_{k_{1}}, r\right)}\left|f\left(w_{1}, \ldots, w_{m}\right)\right|^{p} \\
\vdots \\
w_{m} \in B_{D}\left(a_{k_{m}}, r\right)
\end{array}\right) \\
\\
\cdot\left(\delta\left(a_{k_{1}}\right)\right)^{\tau_{1} / m} \cdots\left(\delta\left(a_{k_{m}}\right)\right)^{\tau_{m} / m} \leq C_{1} J_{1},
\end{aligned}
$$


where

$$
J_{1}=\int_{D} \cdots \int_{D}\left|f\left(w_{1}, \ldots, w_{m}\right)\right|^{p} \prod_{j=1}^{m}\left(\delta\left(w_{j}\right)\right)^{s_{j}} d m\left(w_{j}\right),
$$

where $\tau_{j}=(n+1) m+s_{j} m, j=1, \ldots, m$. We used at the last step the fact that $\left\{B_{D}\left(a_{k}, R\right)\right\}, R=(1 / 2)(1+r)$ family is a finite covering of $D$, and so

$$
\begin{aligned}
\sum_{k_{1} \geq 0} \ldots \sum_{k_{m} \geq 0} \int_{B_{D}\left(a_{k_{1}}, R\right)} & \\
\cdots & \int_{B_{D}\left(a_{k_{m}}, R\right)}\left|f\left(w_{1}, \ldots, w_{m}\right)\right|^{p} \\
& \cdot \prod_{j=1}^{m}\left(\delta\left(w_{j}\right)\right)^{s_{j}} d m\left(w_{j}\right) \leq C J_{1} .
\end{aligned}
$$

A very careful analysis of the proof of Theorem 1 shows that we replaced properties of $D\left(a_{k}, r\right) r$-lattices of the unit ball of $\mathbb{C}^{n}$ (see $[2,4]$ and references therein) with similar properties of $r$-lattice invented recently in strictly pseudoconvex domains in important papers $[5,6]$. This direction of arguments (replacement of $r$-lattices) can be applied practically to all assertions from $[2,4]$; we partially formulated some parallel assertions above. In view of the mentioned similarities, we give now complete proofs of Theorems 12 and 14 leaving proofs of other assertions to readers and referring to arguments from $[2,4]$.

Proof of Theorems 8 and 12. Remark 11 shows that, for any $f \in$ $A_{t}^{p}, 0<p<\infty, t=\sum_{i=1}^{m} \beta_{i}+(m-1)(n+1)$, there is analytic function $F(z, \ldots, z)=f(z), z \in D$ :

$$
F\left(z_{1}, \ldots, z_{m}\right)=\int_{D} f(\xi) \prod_{j=1}^{m} K_{s_{0} / m}\left(z_{j}, \xi\right)(\delta(\xi))^{\tilde{\beta}} d m(\xi),
$$

where $s_{0}=\widetilde{\beta}_{0}+n+1$, for sufficiently large $\widetilde{\beta}$ (by Lemma C and Proposition 7). All we need to show now is that $\|F\|_{A_{\tilde{\beta}}^{p}} \leq$

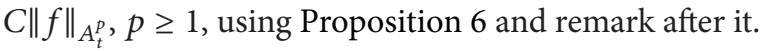

Note that for $p=1$ the assertion is easy to see and follows from Fubini's theorem directly. For $p>1$, we have the following chain of estimates:

$$
\begin{gathered}
\left(\int_{D}|f(\xi)|^{p}(\delta(\xi))^{\tilde{\beta}}\left[\prod_{j=1}^{m}\left|K_{s / m}\left(z_{j}, \xi\right)\right|\right]^{1 / m} d m(\xi)\right)^{p} \\
\leq C_{1}\left(\int_{D}|f(\xi)|^{p}\left(\delta(\xi)^{\tilde{\beta}}\right) \prod_{j=1}^{m}\left|K_{s / m}\left(z_{j}, \xi\right)\right|^{\gamma_{1} p} d m(\xi)\right) \\
\cdot\left(\int_{D}(\delta(\xi))^{\tilde{\beta}} \prod_{j=1}^{m}\left|K_{s / m}\left(z_{j}, \xi\right)\right|^{\gamma_{2} q} d m(\xi)\right)^{p / q}
\end{gathered}
$$

$$
\begin{aligned}
\leq & C_{2}\left(\int_{D}|f(\xi)|^{p}(\delta(\xi))^{\tilde{\beta}} \prod_{j=1}^{m}\left|K_{s / m}\left(z_{j}, \xi\right)\right|^{\gamma_{1} p} d m(\xi)\right) \\
& \cdot\left(\int_{D}(\delta(\xi))^{\tilde{\beta}}\left|K_{s / m}\left(z_{j}, \xi\right)\right|^{\gamma_{2} q m} d m(\xi)\right)^{p / q m},
\end{aligned}
$$

where $1 / p+1 / q=1,1<p<\infty, \gamma_{1}+\gamma_{2}=1, z_{j} \in D$, $j=1, \ldots, m$, and $s=\widetilde{\beta}+n+1$. Hence, we have by preliminaries of the previous section

$$
\begin{aligned}
& \|F\|_{A_{\tilde{\beta}}^{p}} \\
& \leq C_{1} \int_{D} \ldots \int_{D}\left|F\left(z_{1}, \ldots, z_{m}\right)\right|^{p}\left(\delta\left(z_{1}\right)\right)^{\beta_{1}} \\
& \cdots\left(\delta\left(z_{m}\right)\right)^{\beta_{m}} d m\left(z_{1}\right) \cdots d m\left(z_{m}\right) \\
& \leq C_{2} \int_{D} \cdots \int_{D}\left(\delta\left(z_{1}\right)\right)^{\beta_{1}} \cdots\left(\delta\left(z_{m}\right)\right)^{\beta_{m}} \\
& \cdot \int_{D}|f(\xi)|^{p} \cdot(\delta(\xi))^{\tilde{\beta}} \\
& \cdot\left(\prod_{j=1}^{m}\left|K_{s / m}\left(z_{j}, \xi\right)\right|^{\gamma_{1} p}\right)\left(\delta\left(z_{1}\right)\right)^{x_{1}} \\
& \cdots\left(\delta\left(z_{m}\right)\right)^{x_{m}} d m(\xi) d m\left(z_{1}\right) \cdots d m\left(z_{m}\right),
\end{aligned}
$$

where $x_{i}=(\widetilde{\beta}+n+1)(p / q m)-(\widetilde{\beta}+n+1)\left(\gamma_{2} p / m\right), i=1, \ldots, m$, since $\widetilde{\beta}$ is large enough.

An application of Fubini's theorem and the same estimate we just used above lead finally to the estimate that is

$$
\|F\|_{A_{\vec{\beta}}^{p}} \leq C\|f\|_{A_{t}^{p}},
$$

where $p \in(1, \infty), \quad t=\sum_{i=1}^{m} \beta_{i}+(m-1)(n+1)$.

At the final step we used the following inequality in particular:

$$
\begin{gathered}
\prod_{i=1}^{m} \int_{D}\left(\delta\left(z_{i}\right)\right)^{\beta_{i}+(\widetilde{\beta}+n+1)(p / q m)-(\widetilde{\beta}+n+1)\left(\gamma_{2} p / m\right)} \\
\cdot\left|K_{s / m}\left(z_{i}, \xi\right)\right|^{\gamma_{1} p} d m\left(z_{i}\right) \\
\leq C(\delta(\xi))^{(-\widetilde{\beta})+\sum_{i=1}^{m} \beta_{i}+(m-1)(n+1)}, \quad \xi \in D,
\end{gathered}
$$

for certain fixed $\gamma_{1}, \gamma_{2}, \gamma_{1}+\gamma_{2}=1$ and large enough $\widetilde{\beta}$, $1 / p+1 / q=1,1<p<\infty$. Note that an easy technical computation shows that such $\gamma_{1}$ and $\gamma_{2}$ can be found. We leave this inspection to readers.

The theorem is proved. 
Remark 18. Note the assertion we just proved is in a little bit general form and can be found in Theorem 15. We omit details referring the readers to $[2,4]$ where unit ball case was considered.

Remark 19. Note it is easy to see the same arguments are valid if we replace in formulations of our theorems kernels $K_{\alpha / m}$ by $\left|K_{\alpha}\right|^{1 / m}$ to get the same results.

The next lemma, Lemma $\mathrm{D}$, as in unit ball case is playing the crucial role in the proof of Theorem 14 ( $p \leq 1$ case). We however show Theorem 14 using additional assumptions on $K_{t}$ kernel. These assumptions (which are valid in the unit ball also) can be dropped using Lemma $\mathrm{C}$ and this is also completely similar to the proof of the unit ball case.

Lemma $\mathbf{D}$ (see [4]). Let $F \in H(D), p \leq 1, \beta>-1, \alpha$ is a large enough natural number, and $z_{k} \in D, k=1, \ldots, m$. One has the following estimate:

$$
\begin{gathered}
\left(\int_{D}|F(z)| \prod_{k=1}^{m}\left|K_{\alpha}\left(z, z_{k}\right)\right| \delta^{\beta}(z) d m(z)\right)^{p} \\
\leq C \int_{D}|F(z)|^{p} \delta(z)^{\beta p+p(n+1)-(n+1)} \\
\cdot \prod_{k=1}^{m}\left|K_{\alpha}\left(z, z_{k}\right)\right|^{p} d m(z)
\end{gathered}
$$

for some positive constant $C$.

In unit disk this lemma can be seen in [8]. In unit ball this lemma can be seen in [4]. This lemma is crucial for the proof of trace theorems in unit disk and unit ball. Moreover, for $m=1$ case in pseudoconvex domains, we refer the reader to [12].

Proof of Theorem 14. We start with the proof of the first part of our theorem. As in proof of the previous theorem, for every positive large enough $\beta_{j}, j=1, \ldots, n$, we have $F(z, \ldots, z)=$ $f(z)$, where

$$
\begin{aligned}
& F\left(z_{1}, \ldots, z_{m}\right) \\
& \quad=C_{\widetilde{\beta}} \int_{D} f(w)(\delta(w))^{\tilde{\beta}} \prod_{j=1}^{m} K_{s / m}\left(w, z_{j}\right) d m(w),
\end{aligned}
$$

where $C_{\widetilde{\beta}}$ is a Bergman representation constant, for large enough $\widetilde{\beta}, s=\widetilde{\beta}+n+1$.

Note first that it is obvious $\operatorname{Trace}\left(A_{\vec{r}}^{\infty}\right) \subset A_{r}^{\infty}(D)$. Since obviously we have

$$
\begin{aligned}
& \sup _{z \in D}|f(z, \ldots, z)|(\delta(z))^{\sum_{j=1}^{m} r_{j}} \\
& \leq \sup _{z_{1} \in D}\left|f\left(z_{1}, \ldots, z_{m}\right)\right| \prod_{j=1}^{m}\left(\delta\left(z_{j}\right)\right)^{r_{j}}, \\
& \vdots \\
& z_{m} \in D
\end{aligned}
$$

to show the reverse we get by Hölder's inequality from (45) and preliminary estimates of Forelli-Rudin type which we mentioned above

$$
\begin{aligned}
& \left|F\left(z_{1}, \ldots, z_{m}\right)\right| \\
& \leq C_{1}\|f\|_{A_{\vec{r}}^{\infty}} \\
& \quad \cdot \int_{D}(\delta(w))^{\tilde{\beta}-\sum_{j=1}^{m} r_{j}} \\
& \quad \cdot \prod_{j=1}^{m}\left|K_{(\widetilde{\beta}+n+1) / m}\right|\left(w, z_{j}\right) d m(w) \\
& \leq C_{2}\|f\|_{A_{r}^{\infty}} \\
& \quad \cdot \prod_{j=1}^{m}\left(\int_{D}(\delta(w))^{-r_{j} m+\widetilde{\beta}}\right. \\
& \leq \frac{C_{3}}{\left(\delta\left(z_{1}\right)\right)^{r_{1}} \cdots\left(\delta\left(z_{m}\right)\right)^{r_{m}}}, \quad z_{j} \in D, j=1, \ldots, m,
\end{aligned}
$$

by Corollary 3.9 from [12] for large enough $\widetilde{\beta}$ and the first part of Theorem 14 is proved.

We turn now to the proof of the second part of Theorem 14, the case of $A_{\vec{\alpha}}^{p}$ spaces for $p \leq 1$.

The proof follows directly from Theorem 1 (the proof was provided above) and from the third part of Theorem 15. We turn to the proof of the third part of Theorem 15 completing the proof at the same time again using properties of $r$-lattices of Kobayashi ball and we have the following inequalities.

Here we need

$$
\begin{aligned}
\int_{B_{D}\left(a_{k}, r\right)}(\delta(w))^{s} d m(w) & =\left(\delta\left(a_{k}\right)\right)^{n+1+s}, \\
a_{k} & \in D, \quad s>-1 .
\end{aligned}
$$

These facts and Propositions 3-7 above lead us to the following chain of estimates which finish the proof of the inequality we wished to show.

We put additional condition on Bergman kernel below, but with the help of Lemma D it can be removed. Note similar arguments we used in the case of unit ball. We have now using (35)

$$
\begin{aligned}
& \left|\left(S_{\vec{a}, \vec{b}} f\right)\left(\xi_{1}, \ldots, \xi_{m}\right)\right| \\
& \leq C_{1} \sum_{k=1}^{\infty} \prod_{j=1}^{m}\left(\delta\left(\xi_{i}\right)\right)^{\widetilde{a_{i}}} \\
& \quad \cdot \sum_{k=1}^{\infty} \int_{B_{D}\left(a_{k}, r\right)}|f(\vec{z})|(\delta(z))^{-n-1+\sum_{j=1}^{m} b_{j}}
\end{aligned}
$$




$$
\begin{gathered}
\leq \prod_{2} \sum_{k=1}^{\infty}\left|K_{\tau_{i}}\left(\xi_{i}, z\right)\right| d m(z) \\
\left.\cdot \int_{B_{D}\left(a_{k}, r\right)}^{m}\left(\delta\left(\xi_{i}\right)\right)^{\widetilde{a_{i}}}(z)\right)^{-n-1+\sum_{j=1}^{m} b_{j}} \\
\cdot \prod_{z \in B_{D}\left(a_{k}, r\right)}^{m}\left|K_{\tau_{i}}\left(\xi_{i}, z\right)\right| d m(z) \mid \\
\leq C_{3} \sum_{k=1}^{\infty} \prod_{j=1}^{m}\left(\delta\left(\xi_{i}\right)\right)^{\widetilde{a}_{i}} \max _{z \in B_{D}\left(a_{k}, r\right)}|f(z)| \\
\cdot\left(\delta\left(a_{k}\right)\right)^{-n-1+\sum_{j=1}^{m} b_{j}}\left(\delta\left(a_{k}\right)\right)^{n+1} \\
\cdot \prod_{i=1}^{m}\left|K_{\tau_{i}}\left(\xi_{i}, a_{k}\right)\right|,
\end{gathered}
$$

where $\tau_{i}=a_{i}+b_{i}, i=1, \ldots, m$, and $\xi_{i} \in D, i=1, \ldots, m$. Consider

$$
\begin{aligned}
& \left\|\left(S_{\vec{a}, \vec{b}}\right)(f)\right\|_{A_{s_{1}, \ldots, s_{m}}^{p}} \\
& \leq C_{4} \sum_{k=1}^{\infty} \sup _{w \in B_{D}\left(a_{k}, r\right)}|f(w)|^{p}(\delta(w))^{m(n+1)+\sum_{j=1}^{m} s_{j}} \\
& \leq C_{5} \sum_{k=1}^{\infty} \int_{B_{D}\left(a_{k}, r\right)}|f(w)|^{p}(\delta(w))^{(m-1)(n+1)+\sum_{j=1}^{m} s_{j}} d m \\
& \quad \leq C_{6} \int_{D}|f(w)|^{p}(\delta(w))^{(m-1)(n+1)+\sum_{j=1}^{m} s_{j}} d m(w) .
\end{aligned}
$$

The proof of Theorem 14 is finished.

Remark 20. Some results of this paper can be extended to the so-called analytic Herz type spaces in product domains. To define the Herz space based on Kobayashi balls, we remind the reader that there exists a family of Kobayashi balls $B_{\Omega}\left(a_{k}, r\right)$ which forms an $r$-lattice in bounded strictly pseudoconvex domain $\Omega$ (see $[5,6,12])$. We denote by $B_{\Omega}^{m}(z, r) m$ products of such Kobayashi $B_{\Omega}(z, r)$ balls in $\mathbb{C}^{m}$, where $z \in \Omega$. By $M_{v, \tau}^{p}\left(\Omega^{m}\right)$, we denote all $f$ analytic functions in $\Omega^{m}$ so that

$$
\int_{B_{\Omega}^{m}(Z, r)}\left|f\left(z_{1}, \ldots, z_{m}\right)\right|^{p} \prod_{j=1}^{m} \delta^{s_{j}}\left(z_{j}\right) d z_{j}
$$

belongs to $L_{\tau_{1}, \ldots, \tau_{m}}^{1}\left(\Omega^{m}\right)$, where $s_{j}>-1, \tau_{j}>-1$ for all $j=$ $1, \ldots, m, 1 \leq p<\infty$

It will be interesting to extend our results to $A_{\delta, k}^{p, q}$ spaces; some ideas from this paper can be used probably.
Remark 21. Some results of this paper can be also obtained by similar technique in bounded symmetric domains and bounded minimal homogeneous domains in higher dimension.

\section{Conflict of Interests}

The authors declare that there is no conflict of interests regarding the publication of this paper.

\section{Acknowledgment}

Olivera R. Mihić was supported by MNTR Serbia, Project 174017.

\section{References}

[1] M. Arsenović and R. F. Shamoyan, "Trace theorems in harmonic function spaces on $\left(\mathbb{R}_{+}^{n+1}\right)^{m}$, multipliers theorems and related problems," Kragujevac Journal of Mathematics, vol. 35, no. 3, pp. 411-430, 2011.

[2] R. F. Shamoyan and O. R. Mihić, "On traces of holomorphic functions on the unit polyball," Applicable Analysis and Discrete Mathematics, vol. 3, no. 2, pp. 198-211, 2009.

[3] R. F. Shamoyan and O. R. Mihić, "A note on traces of some holomorphic spaces on polyballs," Journal of Function Spaces and Applications, vol. 8, no. 3, pp. 271-285, 2010.

[4] R. F. Shamoyan and O. R. Mihić, "On traces of Qp type spaces and mixed norm analytic function spaces on polyballs," Siauliai Mathematical Seminar, vol. 5, no. 13, pp. 101-119, 2010.

[5] M. Abate and A. Saracco, "Carleson measures and uniformly discrete sequences in strongly pseudoconvex domains," Journal of the London Mathematical Society, vol. 83, no. 3, pp. 587-605, 2011.

[6] M. Abate, A. Saracco, and J. Raissy, "Toeplitz operators and Carleson measures in strongly pseudoconvex domains," Journal of Functional Analysis, vol. 263, no. 11, pp. 3449-3491, 2012.

[7] W. Rudin, Function Theory in Polydisk, W. A. Benjamin, New York, NY, USA, 1969.

[8] F. A. Shamoian and M. M. Djrbashian, Topics in the Theory of Bergman $A_{\alpha}^{p}$ Spaces, Teubner Texte zur Math, Leipzig, Germany, 1988.

[9] M. Jevtić, M. Pavlović, and R. F. Shamoyan, "A note on the diagonal mapping in spaces of analytic functions in the unit polydisc," Publicationes Mathematicae Debrecen, vol. 74, no. 12, pp. 45-58, 2009.

[10] E. Amar and C. Menini, "A counterexample to the corona theorem for operators on $H^{2}\left(\mathbb{D}^{n}\right)$," Pacific Journal of Mathematics, vol. 206, no. 2, pp. 257-268, 2002.

[11] K. Adachi, "Extending bounded holomorphic functions from certain subvarieties of a weakly pseudoconvex domain," Pacific Journal of Mathematics, vol. 110, no. 1, pp. 9-19, 1984.

[12] F. Beatrous, " $L^{p}$ estimates for extentions of holomorphic functions," Michigan Mathematical Journal, vol. 32, pp. 361-380, 1985.

[13] G. M. Henkin, "Continuation of bouded holomorphic functions from submanifolds to strictly pseudoconvex domains," Mathematics of the USSR-Izvestiya, vol. 6, no. 3, pp. 536-563, 1972.

[14] L. I. Ronkin, Introduction to the Theory of Entire Functions of Several Variables, American Mathematical Society, 1974. 
[15] J. M. Ortega and J. Fabrega, "Mixed-norm spaces and interpolation," Studia Mathematica, vol. 109, no. 3, pp. 233-254, 1994.

[16] T. Jimbo and A. Sakai, "Interpolation manifolds for products of strictly pseudoconvex domains," Complex Variables, Theory and Application, vol. 8, no. 3-4, pp. 333-341, 1987.

[17] S. R. Bell, "A representation theorem in strictly pseudoconvex domains," Illinois Journal of Mathematics, vol. 26, no. 1, pp. 1926, 1982.

[18] H. P. Boas, "Sobolev space projections in strictly pseudoconvex domains," Transactions of the American Mathematical Society, vol. 288, no. 1, pp. 227-240, 1985.

[19] W. S. Cohn, "Weighted Bergman projections and tangential area integrals," Studia Mathematica, vol. 106, no. 1, pp. 59-76, 1993.

[20] P. Jakóbczak, "The boundary regularity of the solution of the $\bar{\partial}$-equation in the product of strictly pseudoconvex domains," Pacific Journal of Mathematics, vol. 121, no. 2, pp. 371-381, 1986.

[21] H. R. Cho and J. Lee, "Inequalities for the integral means of holomorphic functions in the strongly pseudoconvex domain," Communications of the Korean Mathematical Society, vol. 20, no. 2, pp. 339-350, 2005.

[22] M. Engliš, T. Hänninen, and J. Taskinen, "Minimal $L^{\infty}$-type spaces on strictly pseudoconvexdomains on which the Bergman projection is continuous," Houston Journal of Mathematics, vol. 32, no. 1, Article ID 253275, 2006. 


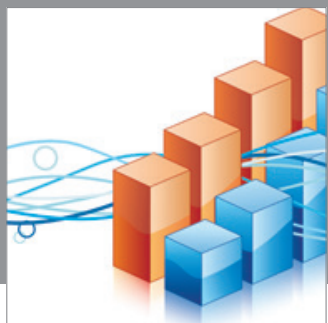

Advances in

Operations Research

mansans

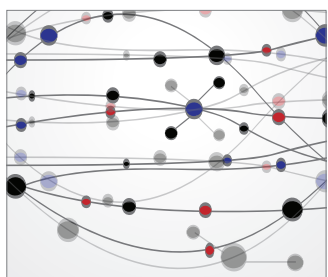

The Scientific World Journal
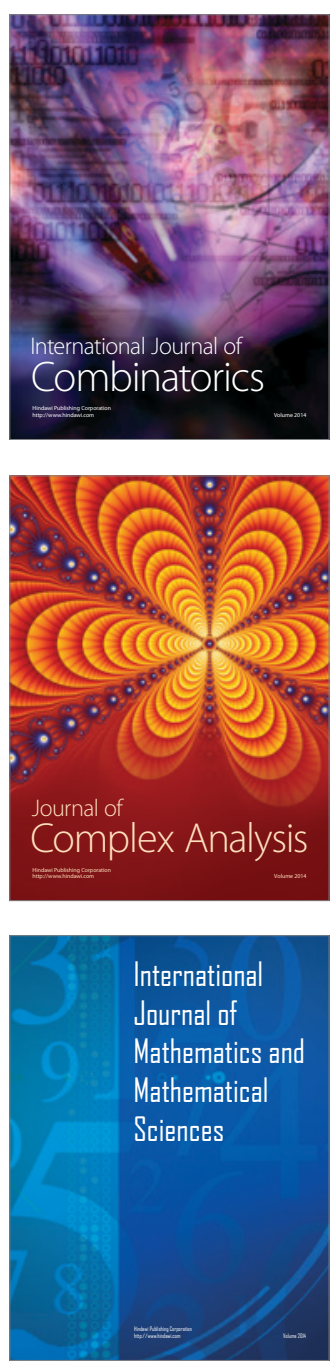
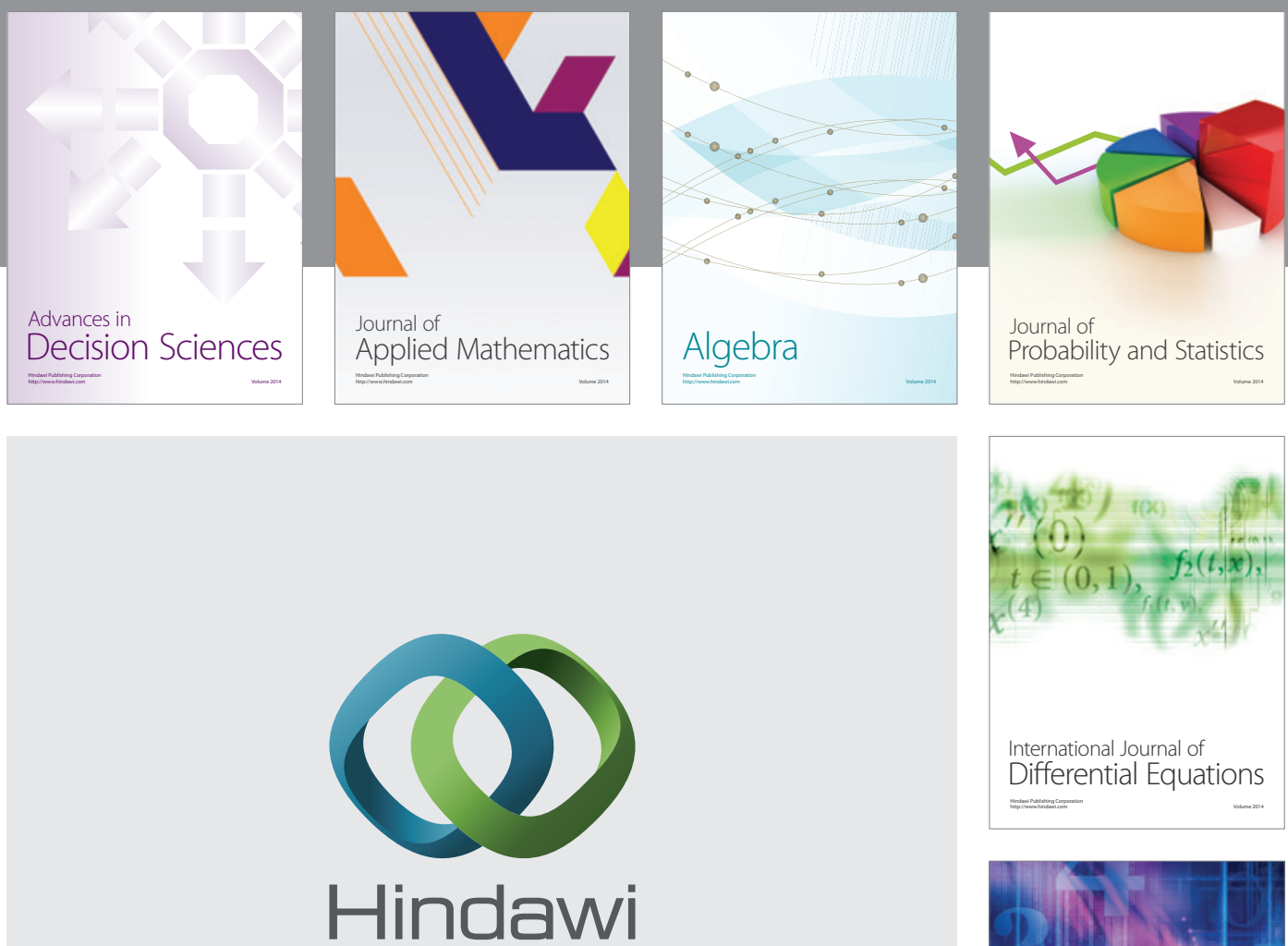

Submit your manuscripts at http://www.hindawi.com
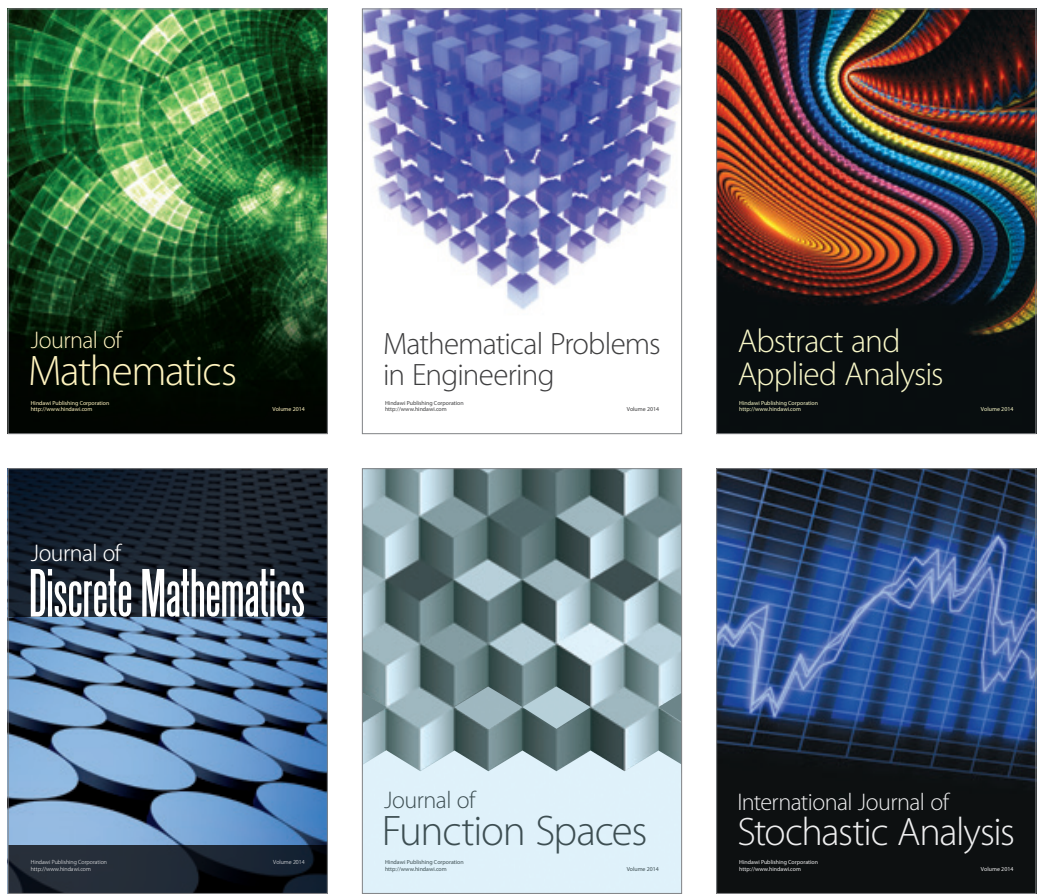

Journal of

Function Spaces

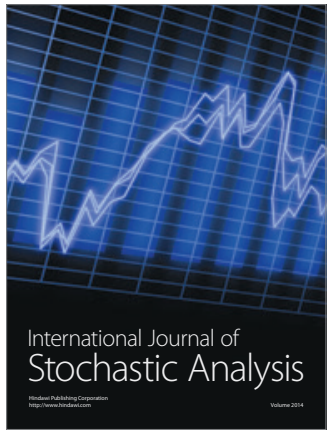

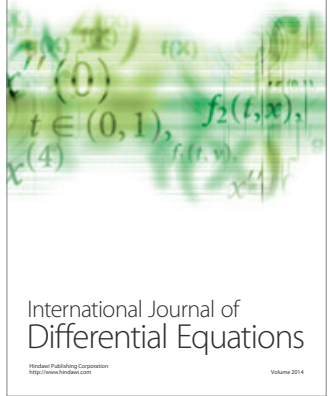
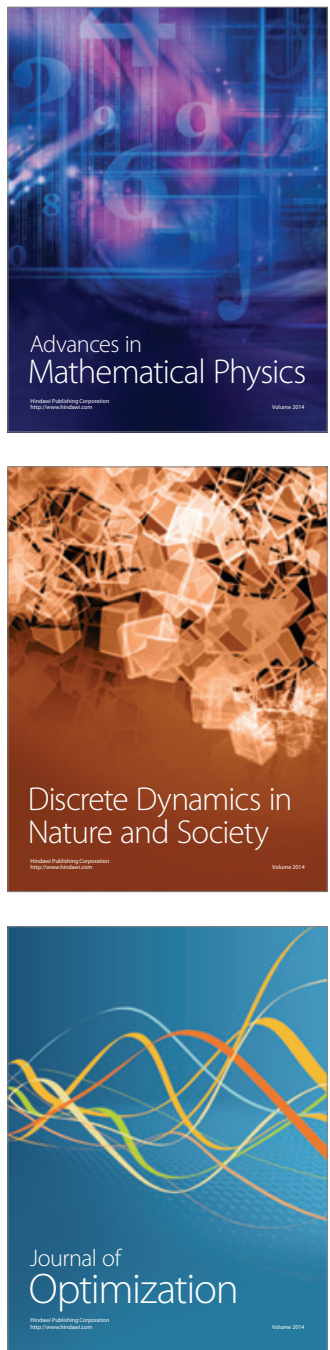